\title{
Insuficiencia cardiaca en el paciente VIH
}

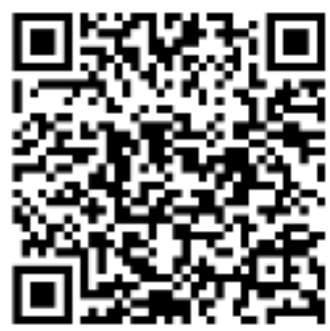

RECIBIDO

$18 / 03 / 2019$

\author{
${ }^{1}$ Dr. Marco Tulio Gómez Cerdas \\ Clínica Carlos Duran Cartin, San José, Costa Rica \\ mgc11@hotmail.com \\ https://orcid.org/0000-0002-5329-3359 \\ ${ }^{2}$ Dra. Silvia Ramírez Hidalgo \\ Clínica Carlos Duran Cartin, San José, Costa Rica \\ silramirezhidalgo@hotmail.com \\ https://orcid.org/0000-0001-6780-8969
}

CORREGIDO ACEPTADO

$27 / 03 / 2019$

$11 / 04 / 2019$

\section{RESUMEN}

La infección por el virus de la inmunodeficiencia humana se ha extendido a todos los rincones del planeta, siendo una de las infecciones más mortíferas del siglo pasado. En esta población se han identificado diversas manifestaciones cardiovasculares, dentro de estas se encuentra la insuficiencia cardiaca. En general, se trata de una complicación tardía de la infección por el virus de la inmunodeficiencia humana. Se han descrito múltiples mecanismos fisiopatológicos implicados en la alteración metabólica y cardiovascular que pueden ocasionar a largo plazo insuficiencia cardíaca. Dentro de las manifestaciones clínicas en los pacientes con virus de la inmunodeficiencia humana se han detectado los signos clásicos de insuficiencia cardiaca congestiva: disnea, fatiga, edemas, entre otros. Actualmente, las estrategias para reducir el riesgo de insuficiencia cardíaca incluyen la instauración temprana de la terapia antirretroviral con mínimos efectos metabólicos posibles y un tratamiento cuidadoso de los factores de riesgo cardiovascular tradicionales.

PALABRAS CLAVE: Insuficiencia cardíaca; VIH; síndrome de inmunodeficiencia adquirida; cardiomiopatías

Médico general, graduado de la Universidad de Iberoamérica (UNIBE). Código médico: 15082 mgc11@hotmail.com.

${ }^{2}$ Médico general, graduada de Universidad Hispanoamericana (UH). Código médico: 13487 silramirezhidalgo@hotmail.com.

\section{ABSTRACT}

The infection by the human immunodeficiency virus has spread to all corners of the planet, being one of the deadliest infections of the last century. In this population, several cardiovascular manifestations have been identified, including heart failure. In general, it is a late complication of human immunodeficiency virus infection. Multiple pathophysiological mechanisms involved in metabolic and cardiovascular disorders that can cause long-term heart failure have 
been described. Among the clinical manifestations in patients with human immunodeficiency virus, the signs of congestive heart failure have been detected: dyspnea, fatigue, edema, among others. Currently, strategies to reduce the risk of heart failure include the early establishment of antiretroviral therapy with minimal possible metabolic effects and careful treatment of traditional cardiovascular risk factors.

KEYWORDS: heart failure, HIV, acquired immunodeficiency syndrome, cardiomyopathies.

\section{INTRODUCCIÓN}

La infección por el virus de la inmunodeficiencia humana $(\mathrm{VIH})$ se ha extendido a todos los rincones del planeta, siendo una de las infecciones más mortíferas del siglo pasado; se estima que hoy en el mundo hay aproximadamente 34 millones de personas que viven con el $\mathrm{VIH}$, y cerca de un $90 \%$ se encuentra en países en desarrollo (1-3).

En 1983 se describe por primera vez el compromiso cardíaco en pacientes con $\mathrm{VIH}$, por la médica Brigitte Autran, quien encontró un sarcoma de Kaposi miocárdico a través de la autopsia. Actualmente, es frecuente encontrar en las autopsias signos de compromiso cardíaco en más del $73 \%$ de los pacientes VIH fallecidos $(2,4)$.

Dentro de las comorbilidades cardíacas más frecuentes asociadas a esta población se encuentran: la cardiopatía isquémica, miocardiopatía dilatada, miocarditis infecciosa y no infecciosa, necrosis miocárdica asociada o no a inflamación y compromiso ventricular derecho dependiente de patología pulmonar (incluyendo compromiso a nivel de vasculatura pulmonar). Además, a nivel valvular se ha observado endocarditis infecciosa o endocarditis marántica; en el pericardio, derrame infectado o no, con o sin taponamiento, y pericarditis fibrinosa o constrictiva. Por último, se ha identificado compromiso neoplásico por sarcoma de Kaposi o linfoma $(2,3,5)$.

Debido al gran avance médico en el diagnóstico oportuno y tratamiento del $\mathrm{VIH}$, se ha presentado un incremento en la expectativa de vida en esta población, ocasionando que la incidencia de enfermedades relacionadas con la edad también aumente, como es el caso de las enfermedades cardiovasculares. Lo anterior se ha asociado a la combinación del envejecimiento, aumento de factores de riesgo tradicionales (edad, hipertensión arterial, tabaquismo, diabetes mellitus), efectos directos del virus y a la respuesta inmunológica del huésped como una consecuencia directa de la infección (principalmente con cifras de linfocitos T CD4+ iniciales $<500 / \mu$; lo que podría vincularse con la activación inmunitaria e incremento a la propensión a la coagulación que se observan como consecuencia de la replicación de VIH) o incluso ser una complicación de la terapia antiretroviral (TAR) $(2,4-6)$.

Antes de la era de la TAR, la cardiomiopatía asociada al VIH se expresó como insuficiencia sistólica con dilatación del ventrículo izquierdo y se 
observó casi exclusivamente en pacientes con enfermedad avanzada por VIH. Este tipo de insuficiencia cardíaca (IC) todavía es común en áreas geográficas donde el tratamiento antirretroviral no está ampliamente disponible. Por el contrario, en la era actual, el diagnóstico de IC incluye muchas más personas con VIH asintomáticas $\mathrm{y}$, a menudo, se refiere solo a la insuficiencia sistólica o diastólica detectada mediante una ecocardiografía (7-9).

En el presente artículo se hará un análisis puntual de la revisión bibliográfica encontrada sobre la IC en la población $\mathrm{VIH}$, determinando su epidemiologia, fisiopatología, diagnóstico y principales recomendaciones terapéuticas que podrían favorecer a esta determinada población.

\section{EPIDEMIOLOGÍA}

En las autopsias de pacientes infectados por $\mathrm{VIH}$ se ha determinado cerca de un $25 \%$ a $75 \%$ hallazgos de alguna enfermedad cardiovascular, entre ellas se encuentran la cardiopatía coronaria, miocardiopatía dilatada y la insuficiencia cardiaca (IC) (5).

La incidencia y prevalencia de IC asociada al VIH en Latinoamérica es difícil de evidenciar ya que hay escaso registro en la evidencia científica. Sin embargo, con la aparición de una mejor TAR ha cambiado que estos pacientes ya no presenten una cardiomiopatía dilatada y severa (antes vista en pacientes con enfermedad avanzada por $\mathrm{VIH}$ y síndrome de inmunodeficiencia adquirida "SIDA") sino una cardiomiopatía con función sistólica del ventrículo izquierdo sintomática; aunque además recientemente se ha observado un aumento significativo con parámetros anormales en la función diastólica, llevando a síntomas de insuficiencia cardiaca (10).

Existen dos estudios observacionales realizados en África Subsahariana, uno fue el HoS (Heart of Soweto) y el otro el THESUS-HF (The Sub-Saharan Africa Survey of Heart Failure) de los cuales indicaron una prevalencia de IC en pacientes $\mathrm{VIH}$ de aproximadamente el $50 \%$ y una incidencia de cualquier anomalía cardíaca del $55 \%$ durante un período de 7 años. La insuficiencia sistólica del ventrículo izquierdo fue la más común entre los pacientes sintomáticos, mientras que los asintomáticos se observó IC con fracción de eyección preservada. La IC ocurrió con mayor frecuencia en personas jóvenes con recuentos de células T CD4 + de $<100$ células / mm3, menor nivel socioeconómico, mayor duración de la infección por $\mathrm{VIH}$, mayor carga viral y estadio avanzado del VIH; además la tasa de mortalidad hospitalaria debida a la IC asociada al VIH alcanzó el 15\% $(6,11)$.

El estudio HoS es el registro más grande y completo con presentaciones de novo de cualquier enfermedad cardíaca en la África Subsahariana. Dentro de los 5,328 pacientes con manifestaciones cardíacas entre 2006 y 2008, hubo 518 personas con $\mathrm{VIH}(9,7 \%)$. La mayoría de estos (54\%) tomaban TAR, eran jóvenes, tenían presión arterial óptima, aumento de la frecuencia cardíaca e índice de masa corporal más bajo que el resto del estudio HoS. En un 38\% se detectó IC asociada al $\mathrm{VIH}$ siendo el diagnóstico más frecuente, estos presentaron una 
fracción de eyección del ventrículo izquierdo promedio del $46 \%(6,11)$.

Casi un tercio de los pacientes VIH con IC en el estudio HoS presentó insuficiencia sistólica del ventrículo izquierdo ( $\mathrm{N}=148 ; 29 \%)$ y 196 (38\%) tenía cardiomiopatía relacionada con el VIH (que incluía tanto disfunción sistólica como diastólica en pacientes sintomáticos y asintomáticos). Se estimó que las mujeres sufren más de IC asociada al VIH que en comparación a los hombres, La carga viral fue significativamente mayor $(110,000$ frente a 19,000 copias de ARN / ml), y los recuentos de células $\mathrm{T}$ CD4 + fueron significativamente más bajos (180 vs. 211 células / mm3) en pacientes con IC asociada al VIH que en la población con el VIH sin IC (6).

El estudio THESUS-HF fue un registro prospectivo multinacional de pacientes con IC descompensada aguda ingresados en hospitales universitarios de 9 países africanos, entre las principales causas de ingreso por IC se determinó: VIH, la cardiopatía reumática, la pericarditis, hipertensión arterial y la cardiopatía isquémica. De los 1.006 pacientes inscritos, el $50 \%$ se sometió a pruebas de detección del VIH y se detectó que el $65 \%$ de estos estaban infectados con VIH (6).

EI VIH fue la causa de la IC en el 2,6\% de todos los casos. La mayoría de estos pacientes eran de Mozambique, Camerún y Uganda, países que también tienen altas tasas de VIH. En esa población se diagnosticó IC desde la tercera década de vida y es de mayor frecuencia en mujeres. Además, las disfunciones sistólicas como las diastólicas fueron las más comunes
( $30 \%$ de prevalencia) y en raras ocasiones, la disfunción diastólica fue la más frecuente (6).

\section{FISIOPATOLOGÍA}

Se han descrito múltiples mecanismos fisiopatológicos implicados en la alteración metabólica y cardiovascular que pueden llegar a ocasionar a largo plazo IC en la población VIH. El curso de la enfermedad de la IC en esta población que no recibe TAR, se desarrolla durante semanas o meses, siendo lo contrario en los pacientes que reciben TAR los cuales cursan sintomáticos de una forma más prolongada. Los mecanismos fisiopatológicos que se han descrito hasta el día de hoy son: toxicidad viral directa, infecciones oportunistas, respuesta del sistema inmune a la infección viral, cardiotoxicidad por medicamentos, deficiencias nutricionales e inmunosupresión prolongada, además se presentan con alta prevalencia factores de riesgo tradicionales de hipertensión, tabaquismo, dislipidemia, diabetes y el síndrome metabólico. Por último, en los últimos años la investigación se ha dirigido a la comprensión del papel del sistema inmune y la inflamación en una compleja interacción de factores aún en estudio $(2,7,12)$.

Se ha determinado en varios estudios científicos que las células miocárdicas y de la corteza cerebral pueden actuar como reservorio del virus por extensos períodos, aún luego de la TAR, induciendo una liberación crónica de citoquinas citotóxicas (factor de necrosis tumoral $\alpha$, interleuquina $1,6,10 \mathrm{y}$ endotelina 1, causando daño progresivo 
del tejido provocando miocardiopatía y encefalopatía. Además, hay hallazgos en estudios in vitro de miocitos cardiacos tanto humano como en roedores donde el virus puede entrar a través de vías independientes de los receptores CCR5 o CXCR4 $(2,12)$.

El daño neuronal, especialmente la alteración del sistema nervioso autónomo potencia el daño funcional de las células miocárdicas por aumento de la actividad adrenérgica con mayor consumo de oxígeno y la disminución de receptores beta adrenérgicos con disminución de la contractilidad. Es por esto, que los pacientes con encefalopatía tienen mayor mortalidad por insuficiencia cardíaca congestiva (ICC) $(2,12)$.

En un análisis del estudio SMART (Strategic of Managment for Antiretroviral Therapy) se analizó el valor predictivo de la proteína C-reactiva, Interleuquina (IL) 6 y dímero-D en relación con la morbimortalidad cardiovascular, concluyendo que existía una asociación directa entre estos marcadores con un mayor riesgo de eventos cardiovasculares, independientemente de otros factores de riesgo. Estudios posteriores mostraron que la interrupción de la TAR coincidía con un aumento significativo en los niveles séricos de IL- 6 y dímero $D(12)$.

Otro mediador de la inflamación relevante en los últimos años es el CD14 soluble; un biomarcador de la activación de monocitos y macrófagos que se ha relacionado con aumento en la mortalidad cardiovascular y que en pacientes con VIH se encuentra aumentado. Igualmente, el CD163 soluble, expresado por macrófagos en las placas ateroescleróticas, se correlaciona con el grado de inflamación arterial y se ha considerado como un potencial marcador de inflamación endotelial en pacientes con VIH (12).

Otro mecanismo inflamatorio que se ha descrito en la literatura es la lesión sobre las células endoteliales, el cual se genera por la expresión de quimiocinas y proteínas de adhesión celular tales como ICAM-1, VCAM-1 y E-selectina que promueven el reclutamiento de monocitos y entrada de células inflamatorias en el endotelio lo cual aumenta el riesgo de eventos cardiovasculares (12).

La autoinmunidad cardiaca hipótesis estudiada desde los años 90 por Currie y Cols., encontraron autoanticuerpos cardiacos circulantes específicos (anticuerpo anti a miosina) en más del $30 \%$ de los pacientes con miocardiopatía por HIV, hallazgos que sugieren que la autoinmunidad es en parte responsable de la enfermedad cardíaca relacionada al virus, y que los anticuerpos podrían ser marcadores de insuficiencia ventricular izquierda $(2,12,13)$.

La IC relacionada a pacientes $\mathrm{VIH}$, se produjo con mayor frecuencia en personas con un conteo menor a 100 células por mm3 de CD4, así mismo se presentó en pacientes con un nivel socioeconómico menor, en etapas avanzadas de la enfermedad y pacientes con cargas virales altas. Una hipótesis implica la toxicidad miocárdica directa viral, donde el ácido ribonucleico del VIH en altas concentraciones, es decir más de 500 copias por $\mathrm{ml}$ de sangre, se asocia con aproximadamente 2,5 veces más probabilidad de desarrollar IC en comparación con individuos no infectados (12). La expresión transgénica de miocardio a través del activador de la transcripción del VIH (proteína Tat), 
activa las células endoteliales, provocando así la disfunción sistólica ventricular izquierda, generando hipertrofia ventricular izquierda y la expresión continua de péptidos natriuréticos perpetuados que dan lugar a un compromiso hemodinámico difuso (12).

Las infecciones secundarias a la inmunosupresión por el HIV pueden causar daño cardíaco, como por ejemplo: miocardiopatía (toxoplasma gondii, citomegalovirus, coxsackie, Epstein Barr y adenovirus), derrame pericárdico (Bacterias: Staphylococcus, Streptococcus, Proteus, Nocardia, Pseudomonas, Klebsiella, Enterococcus, Listeria, Mycobacterias: Mycobacterium tuberculosis, Mycobacterium avium intracellulare, Mycobacterium kansaii, Virus: HIV, herpes simplex 1 y 2, citomegalovirus, otros patógenos: criptococcus, toxoplasma e histoplasma (2).

La desnutrición es común en los pacientes con $\mathrm{VIH}$, especialmente en estadios avanzados de la enfermedad, lo que contribuye a aumentar el riego de disfunción ventricular. Además, se ha observado deficiencia de oligoelementos, selenio (Enfermedad de Keshan), vitamina B12, carnitina, hormona de crecimiento, hormonas tiroideas, todas asociadas al desarrollo de miocardiopatías $(2,8,13)$.

El alto consumo de alcohol en algunos pacientes VIH también puede contribuir a IC. Al igual que en los informes recientes, más del $40 \%$ de los infartos de miocardio (IAM) reportados en el paciente $\mathrm{VIH}$ son en realidad de tipo II o relacionados con la demanda de oxígeno en lugar de ser causados

por

enfermedad aterotrombótica real. Es posible que el abuso de alcohol no reconocido y / o el abuso de cocaína o metanfetamina contribuya a la cardiomiopatía en el VIH (7).

Desde la introducción de la TAR en países desarrollados ha existido una reducción en la incidencia de IC en pacientes $\mathrm{VIH}$ en un $30-50 \%$, aunque la cardiotoxicidad por la TAR es controversial, muchas de las alteraciones cardíacas observadas en pacientes $\mathrm{VIH}$ son secundarias a esta $(4,12)$.

Los análogos de la Timidina (Zidovudina y Estavudina, un inhibidor de la transcriptasa inversa) y la Didanosina se asocian con destrucción difusa de la ultraestructura mitocondrial y con la inhibición de la replicación del ADN mitocondrial. La acidosis láctica producida por la disfunción mitocondrial empeora la función de las células miocárdicas. Estudios recientes evidencian relación entre la utilización de Zidovudina y disfunción diastólica. Estos hallazgos son de suma importancia para los países de bajos y medianos ingresos donde la Zidovudina es todavía una opción de primera línea para el tratamiento del VIH para adultos, adolescentes y niños $(2,13)$.

Más recientemente la introducción de HAART (Highly Active Antiretroviral Therapy) se asoció a lipodistrofia / lipoatrofia, hiperlipidemia, insulinoresistencia y consiguiente aumento de las complicaciones cardiovasculares. Este efecto se debe a la similitud de la región catalítica de la HIV proteasa (blanco de los inhibidores de las proteasas (IP) con dos proteínas humanas que regulan el metabolismo de los lípidos: la proteína 1 ligadora del 
ácido retinoico citoplasmático (CRABP-1) y la proteína relacionada con el receptor de lipoproteína de baja densidad (LRP) $(2,13)$.

Paul W. Hruz en sus investigaciones ha demostrado que el uso clínico de inhibidores de proteasas está asociado con insulinorresistencia y otros cambios metabólicos, que impiden la captación de la glucosa por el músculo cardíaco y aumentan el riesgo cardiovascular a largo plazo. En investigaciones con modelos de ratones transgénicos con miocardiopatía dilatada se demostró que la exposición de Ritonavir, Atazanavir y Lopinavir precipitó IC aguda descompensada y muerte de los ratones transgénicos con edema pulmonar agudo $(13,14)$.

Las dosis altas y prolongadas de interferón alfa utilizadas en el sarcoma de Kaposi pueden producir disfunción miocárdica en pacientes con HIV. Debido a que no se observa el mismo efecto en pacientes sin HIV, el fármaco parecería tener una acción sinérgica con el virus $(2,13)$.

La doxorubicina administrada para el sarcoma de Kaposi y el linfoma no hodking $y$ el foscarnet para el citomegalovirus, pueden producir miocardiopatía dilatada dosis dependiente. La anfotericina B, el ganciclovir, la trimetoprimasulfametoxazol y la pentamidina pueden causar arritmias (taquicardia ventricular, fibrilación ventricular, puntas de torsades por prolongación del QTc, anormalidades de la conducción auriculovertricular) $(2,13)$.

A pesar de encontrarse afectación cardiovascular con esos medicamentos, la actual evidencia sugiere que los tipos específicos de TAR se asocian con un menor riesgo de desarrollar IC. En una cohorte de 21435 personas que viven con el VIH / SIDA en el sistema de asuntos de veteranos, 438 incidentes de insuficiencia cardiaca ocurrieron durante una mediana de seguimiento de 5,4 años. El riesgo de IC fue notablemente más bajo en los pacientes que actualmente usan el tenofovir disoproxil fumarato (TDF) (cociente de riesgo 1/6,68; IC del 95\%: 0,53 a 0,86) en comparación con los pacientes que nunca la usaron. La especulación sobre por qué el TDF podría reducir el riesgo de IC incluye la posibilidad de que mejore el control viral y, por lo tanto, reduzca la inflamación, o que pueda estar relacionado con el efecto hipolipemiante del TDF. Se necesitan estudios adicionales para confirmar este efecto beneficioso del TDF en la IC (7). La enfermedad de las arterias coronarias es un factor importante que contribuye a la IC en las personas que viven con el VIH / SIDA, al igual que en otras poblaciones. Los sobrevivientes de infarto de miocardio con infección por VIH son en promedio una década más joven en comparación con los pacientes no infectados después del infarto de miocardio $y$, por lo tanto, tienen una mejor supervivencia a corto plazo, ya que la edad es un predictor importante de la mortalidad después del infarto de miocardio. Sin embargo, en un análisis por edades y por sexo de una gran base de datos francesa contemporánea, las hospitalizaciones por IC fueron más frecuentes durante un año de seguimiento en pacientes infectados por VIH en comparación con pacientes no infectados (7). 
FIGURA 1. La infección por VIH, a través de varios mecanismos conocidos como viremia, inflamación, coinfecciones y factores de riesgo tradicionales como dislipidemia, tabaquismo y abuso de sustancias, conlleva un mayor riesgo de enfermedad arterial coronaria, enfermedad microvascular y disfunción autonómica / neurohormonal. Posteriormente, estas anomalías, junto con otros mecanismos desconocidos, dan como resultado una enfermedad miocárdica y una disfunción dinámica del ventrículo izquierdo, que a su vez aumentan el riesgo de IC y arritmia.

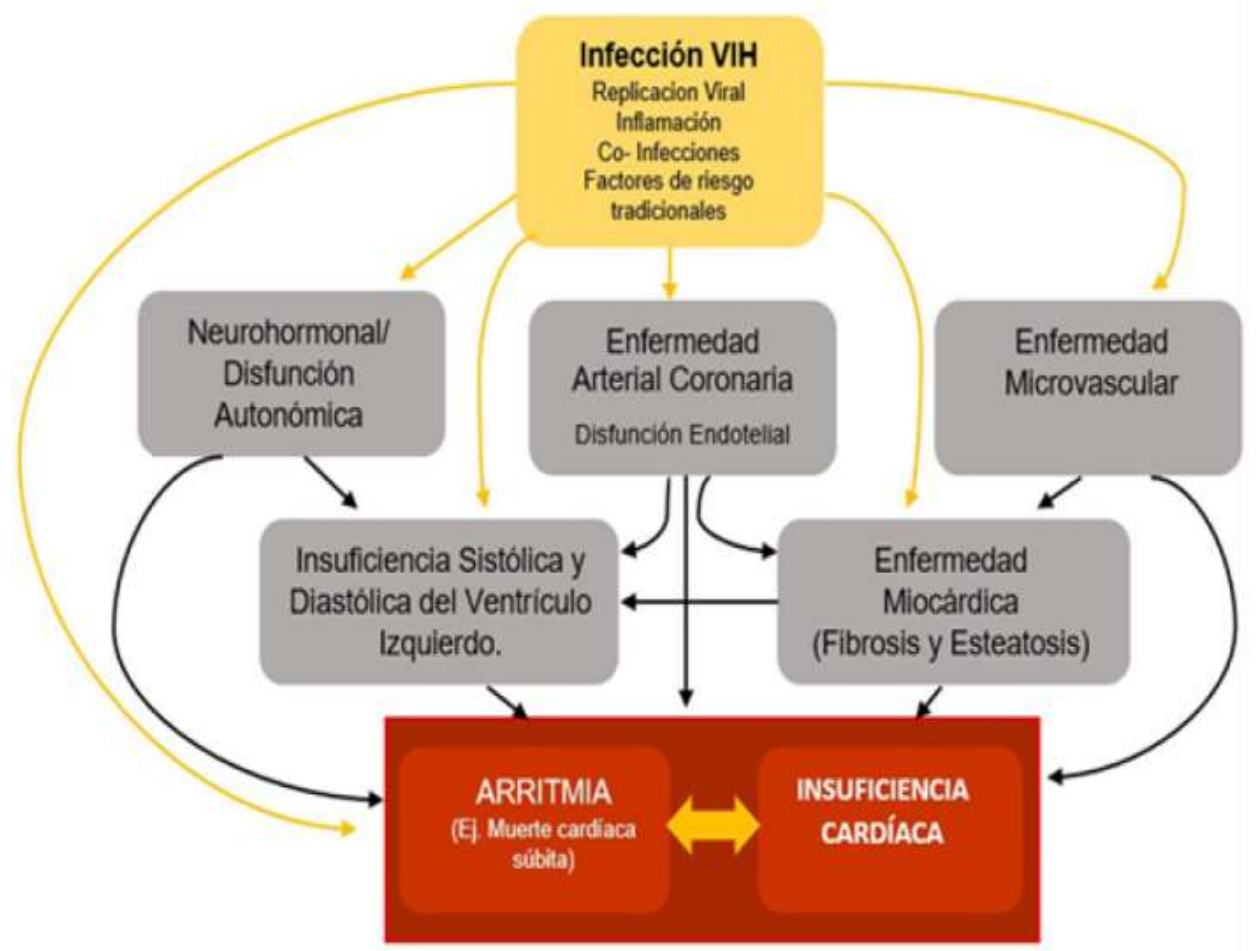

Fuente: figura 1 traducida del articulo HIV Infection and Risk of Cardiovascular Diseases Beyond Coronary Artery Disease (9).

En un análisis reciente, la prevalencia de diabetes mellitus e hipertensión, dos de los factores de riesgo más comunes y reconocidos para la enfermedad coronaria, se encontraron con menos frecuencia en pacientes infectados por el $\mathrm{VIH}$ que sufrieron un infarto agudo de miocardio (IAM) en comparación con los pacientes con IAM que no estaban infectados con el VIH. Sin embargo, la presencia de diabetes confirió un riesgo casi 5 veces mayor de desarrollar IC, al igual que la presencia de la infección por VIH en sí. Se ha determinado en estudios previos que los altos niveles de proteína $\mathrm{C}$ reactiva de alta sensibilidad, el tabaquismo activo y los antecedentes de infarto de miocardio se asociaron significativamente con la insuficiencia sistólica, mientras que la edad avanzada y la hipertensión se relacionaron con un mayor riesgo de insuficiencia diastólica (8).

Finalmente, aunque los pacientes $\mathrm{VIH}$ positivo que reciben TAR no expresan una miocardiopatía manifiesta, los estudios han demostrado un aumento de la disfunción mecánica subclínica cardíaca (tanto sistólica como diastólica) y la presencia de anomalías miocárdicas (fibrosis y esteatosis). La historia natural de estos cambios aún no se ha estudiado, pero sí se ha descrito que 
estos procesos son progresivos y pueden llevar a un riesgo aún mayor de IC a medida que la población infectada con VIH envejece. LA FIGURA 1 proporciona un paradigma general para los mecanismos asociados con un mayor riesgo de $\mathrm{IC}$ basada en el estado actual de nuestro conocimiento. Es importante destacar que se cree que muchas de las mismas vías están involucradas en la causa de las arritmias cardíacas (9).

\section{DIAGNÓSTICO}

Algunos pacientes antes de presentar manifestación de los síntomas pueden cursar con anomalías cardíacas estructurales o funcionales asintomáticas (disfunción sistólica o diastólica del ventrículo izquierdo), que son precursoras de la IC. La identificación de estas anomalías es importante porque se relacionan con peores resultados, y la instauración de tratamiento en esta fase podría reducir la mortalidad de los pacientes con disfunción sistólica ventricular izquierda asintomática (15).

Dentro de las manifestaciones en los pacientes con $\mathrm{VIH}$ se han detectado los signos clásicos de insuficiencia cardíaca congestiva, es decir, disnea, fatiga, que puede ir acompañado de signos como presión venosa yugular elevada, crepitantes pulmonares, edema periférico, reflujo hepatoyugular, entre otros $(5,15)$.

La concentración plasmática de péptidos natriuréticos (PN) puede emplearse como prueba diagnóstica inicial, especialmente en un contexto no agudo cuando no se dispone inmediatamente de ecocardiografía (15).
El límite superior normal en el contexto no agudo para péptidos natriuréticos de tipo B (BNP) es de $35 \mathrm{pg} / \mathrm{ml}$ y para la fracción aminoterminal del propéptido natriurético de tipo B (NT-pro- BNP) es de $125 \mathrm{pg} / \mathrm{ml}$; en el contexto agudo deben emplearse títulos más altos (BNP $<100 \mathrm{pg} / \mathrm{ml}$, NT-proBNP < 300 pg/ml y región media del propéptido natriurético auricular de tipo A [MR-proANP] < 120 $\mathrm{pmol} / \mathrm{l})$. En los umbrales mencionados, los valores predictivos negativos son muy similares y altos $(0,94-0,98)$, tanto en agudos como en no agudos, pero los valores predictivos positivos son más bajos en no agudos $(0,44-0,57)$ y en agudos $(0,66-0,67)$. Por lo tanto, el uso de PN se recomienda para descartar la IC, pero no para establecer el diagnóstico (15).

Los pacientes con concentraciones plasmáticas de PN normales probablemente no tengan IC, sin embargo, la elevación de PN puede producirse por numerosas causas, cardiovasculares y no cardiovasculares (fibrilación auricular, edad, insuficiencia renal), que reducen su capacidad diagnóstica en la IC (15).

Un electrocardiograma (ECG) anormal aumenta la probabilidad del diagnóstico de IC, pero esta prueba tiene una especificidad baja. Algunas anomalías en el ECG dan información sobre la etiología ( $p$. ej., infarto de miocardio) y algunos hallazgos electrocardiográficos pueden proporcionar indicaciones para el tratamiento (p. ej., anticoagulación para la FA, marcapasos para la bradicardia) (15).

La ecocardiografía es la prueba más útil para establecer el diagnóstico en pacientes con sospecha de IC. Esta técnica proporciona información 
inmediata sobre el volumen de las cámaras, la función sistólica y diastólica del VI, el grosor de la pared, la función valvular y la hipertensión pulmonar. Esta información es crucial para establecer el diagnóstico y determinar el tratamiento más adecuado. La información obtenida en la evaluación clínica detallada y con las pruebas antes mencionadas permitirá establecer un diagnóstico inicial y un plan de tratamiento para la mayoría de los pacientes. Generalmente, solo se requieren otras pruebas en caso de que el diagnóstico sea incierto (p. ej., si las imágenes ecocardiográficas no son óptimas o si se sospecha de una causa inusual para la IC) (15).

\section{TRATAMIENTO}

Hoy en día la prevención de las enfermedades cardiovasculares es parte fundamental del tratamiento habitual de los individuos con infección por el $\mathrm{VIH}$. Se debe reducir los factores de riesgo cardiovascular siempre que sea posible. Las estrategias actuales para reducir el riesgo de IC incluyen la instauración temprana de pautas de TAR que tengan los mínimos efectos metabólicos posibles y un tratamiento cuidadoso de los factores de riesgo cardiovascular tradicionales mediante tratamientos médicos y no médicos (1).

Las recomendaciones de tratamiento se basan con estudios realizados en pacientes con IC no infectados y en las directrices basadas en estos estudios, ya que se cuenta con escasos estudios sobre este tema en específico. Por lo tanto, se pueden utilizar los inhibidores de la enzima convertidora de angiotensina, los bloqueadores beta y los antagonistas de la aldosterona, a pesar de que no se han realizado ensayos de estos fármacos en las personas que $\mathrm{VIH}$ con IC $(7,16)$.

Si bien no hay informes sobre los beneficios específicos de los inhibidores de la enzima convertidora de angiotensina y los bloqueadores de renina angiotensina en Ia IC del paciente $\mathrm{VIH}$, un estudio piloto ha sugerido que los bloqueadores de renina angiotensinaaldosterona (telmisartán) podría aumentar las células progenitoras endoteliales y mejorar la reparación vascular en pacientes mayores con $\mathrm{VIH}$ (edad media, 60 años) (9).

Los fármacos antihipertensivos y en especial los antagonistas del calcio dihidropiridinicos pueden interaccionar con los inhibidores de proteasa y deben administrarse a dosis bajas. El ácido acetilsalicílico para la prevención primaria se debe prescribir a los pacientes de riesgo elevado (p. ej., con una puntuación de riesgo de Framingham $\geq 20 \%$ e hipertensión controlada), tal como se recomienda para la población general (1).

Otro de los fármacos utilizados en la IC, es la digoxina la cual es altamente activa contra el $\mathrm{VIH}-1$ in vitro y si esto se valida en estudios en humanos, podría ser una opción atractiva en pacientes $\mathrm{VIH}$ (9).

La TAR no es una terapia específica para la IC; sin embargo, la incidencia de esta patología disminuyó drásticamente después de la introducción de la TAR. Se desconoce si la TAR puede revertir la IC causada por una miocardiopatía establecida. Por otro lado, los medicamentos antirretrovirales, como el Zidovudina, tienen una toxicidad directa al miocardio, y el tratamiento 
antirretroviral puede acelerar la aterosclerosis coronaria, lo que finalmente conduce a una disfunción del ventrículo izquierdo (7).

El papel de la inflamación y la respuesta inmunitaria de la IC asociada al VIH se destaca en un estudio realizado con 49 niños infectados por VIH con dilatación del ventrículo izquierdo, en el cual se observó una mejor contractilidad del ventrículo izquierdo y la disminución de la pared del ventrículo izquierdo en la tensión máxima, en aquellos que se utilizaron niveles altos de inmunoglobulina intravenosa. Sin embargo, a pesar de la ausencia de ensayos específicos con el uso de inmunoglobulina en adultos con $\mathrm{VIH}$, el uso de las mismas guías y terapias de inmunoglobulina para tratar la ICC parece razonable (7-9).

Se cree que el beneficio terapéutico de la inmunoglobulina intravenosa resulta de su capacidad para inhibir la producción de TNF (Factor de necrosis tumoral, del inglés tumor necrosis factor) y la interleucina. Etanercept, otro agente modulador inmune se ha usado en un estudio pequeño de pacientes con IC con éxito moderado. En un estudio en animales, los monos infectados con el virus de inmunodeficiencia de simios y las bacterias inactivas del complejo Mycobacterium avium desarrollaron una disfunción grave del ventrículo izquierdo. Los monos tratados con etanercept no desarrollaron cardiomiopatía, lo que sugiere no solo que el TNF puede desempeñar un papel causal en el desarrollo de la cardiomiopatía por VIH, sino que la terapia dirigida contra TNF también puede tratar la cardiomiopatía. Sin embargo, esta terapia no se ha probado en pacientes humanos infectados con VIH-1 (8).

Se sabe poco sobre el efecto de la terapia con dispositivos cardíacos en pacientes con cardiomiopatía por $\mathrm{VIH}$, sin embargo, también deben considerarse su colocación si lo ameritan. Desafortunadamente, se ha sugerido que los pacientes infectados pueden tener menos probabilidades de recibir un desfibrilador implantable o una terapia de resincronización cardíaca debido a la creencia de que tienen una mayor mortalidad y, por lo tanto, una vida más corta o por temor a complicaciones infecciosas (7-9).

La infección por VIH generalmente se considera una contraindicación para el trasplante cardíaco debido a una supervivencia históricamente pobre y las preocupaciones sobre la progresión al SIDA con inmunosupresión, a pesar de la evidencia reciente que sugiere que los medicamentos inmunosupresores en realidad pueden aumentar la eficacia de la TAR en el tratamiento de la infección por VIH. La evidencia actual indica que el trasplante cardíaco es seguro en pacientes VIH seleccionados, siendo sus resultados similares a los observados en no infectados. En una encuesta de programas de trasplante cardíaco reveló que el $70 \%$ consideró la infección por VIH como una contraindicación absoluta para el trasplante. De hecho, los primeros informes de trasplante cardíaco en pacientes VIH después del trasplante mostraron malos resultados. Sin embargo, desde 2003, cuando se realizó el primer trasplante cardíaco en un paciente $\mathrm{VIH}$, los resultados generalmente han sido favorables. No se ha informado ningún aumento en el rechazo o empeoramiento del estado de 
VIH con inmunosupresión. Las series de casos más grandes en los EE. UU. Y Europa han mostrado resultados similares. Por lo tanto, se hicieron pedidos para reevaluar la infección por VIH-1 como una contraindicación absoluta $(7,8,17)$.

\section{CONCLUSIÓN}

La insuficiencia cardíaca asociada al VIH es causada por una compleja interacción de factores fisiopatológicos relacionadas con el virus, el huésped y la TAR. Es necesario tener un adecuado conocimiento de estos mecanismos o factores para establecer intervenciones médicas individualizadas y así evitar su progresión.
Con la revisión realizada de la literatura sobre la ICC en el paciente VIH se determinó que conforme avancen los nuevos tratamientos de TAR, los factores de riesgo para desarrollar esta patología se asociarán cada vez más a los factores de riesgo cardiovascular tradicionales; por lo que el riesgo de ICC en esta población se espera que aumente para los próximos años. Por lo tanto, es de suma importancia implementar medidas dirigidas a la prevención y modificación de estos factores de riesgo cardiovascular al plantear nuevas estrategias de tamizaje e intervenciones terapéuticas dirigidas a reducir y prevenir los factores implicados para así realizar un adecuado abordaje integral e individualizado.

\section{REFERENCIAS}

1. Boccara F, Cohen A. VIH y Cardiopatía: ¿qué deben saber los cardiólogos?. Rev Esp Cardiol. 2016 Agosto 21; 69(12): 1126-1130. https://doi.org/10.1016/..recesp.2016.05.037

2. Risso G. Enfermedad cardiovascular en sujetos con VIH / SIDA. Rev Fed Arg Cardiol. 2012 Diciembre 7; 41(4): $\quad 235-248 . \quad$ Disponible en http://www.imbiomed.com/1/1/articulos.php?method=showDetail\&id articulo=89388\&id seccion=4471 \&id ejemplar $=8788 \& i d$ revista $=289$

3. Yovanovich S. J. Sindrome de inmunodeficiencia adquirida. compromiso del corazón y de los vasos. Revista Médica Clínica Las Condes. 2015 03; 26(2): 234-240.https://doi.org/10.1016/j.rmclc.2015.04.012

4. Valenzuela G. Patología cardiovascular en pacientes con infección por el virus de inmunodeficiencia humana. An Fac med. 2012 Julio; 73(4): 315-320. Disponible enhttp://www.scielo.org.pe/pdf/afm/v73n4/a08v73n4.pdf

5. Kasper D, Fauci A, Hauser S, Longo D, Jameson J, Loscalzo J. Harrison. Principios de Medicina Interna. McGraw-Hill. 19 edición. 2016. 1255p.

6. Bloomfield G, Alenezi F, Barasa F, Lumsden R, Mayosi B, Velazquez E. Human Immunodeficiency Virus and Heart Failure in Low- and Middle-Income Countries. JACC Heart Fail. 2015 Agosto; 3(8): 579590. https://doi.org/10.1016/j.jchf.2015.05.003

7. Hsue PY, Waters DD. Heart failure in persons living with HIV infection. Current Opinion in HIV and AIDS. 2017 Nov; 12(6): 534-539. https://doi.org/10.1097/coh.0000000000000409

8. Remick J, Georgiopoulou V, Marti C, Ofotokun I, Kalogeropoulos A, Lewis W, et al. Heart Failure in Patients with Human Immunodeficiency Virus Infection: Epidemiology, Pathophysiology, Treatment, and 
Future Research. Circulation. $2014 \quad$ Abril $29 ; \quad 129(17):$ 17811789.https://doi.org/10.1161/CIRCULATIONAHA.113.004574

9. Barnes R, Lacson J, Bahrami H. HIV Infection and Risk of Cardiovascular Diseases Beyond Coronary Artery Disease. Curr Atheroscler Rep. 2017 Mayo; 19(5): 20. https://doi.org/10.1007/s11883-017-0652-3

10. Al-Kindi S, ElAmm C, Ginwalla M, Mehanna E, Zacharias M, Benatti R, et al. Heart failure in patients with human immunodeficiency virus infection: epidemiology and management disparities. Int J Cardiol. 2016 Septiembre 1; 218: 43-46. https://doi.org/10.1016/.ijicard.2016.05.027

11. Manga P, McCutcheon K, Tsabedze N, Vachiat A, Zachariah D. HIV and Nonischemic Heart Disease. JACC. 2017 Enero; 69 (1): 83-91. https://doi.org/10.1016/.jacc.2016.09.977

12. Camargo W, Galvis S, Gualdrón E, Palmezano J. Enfermedad cardiovascular y metabólica en pacientes infectados por el virus de la inmunodeficiencia humana. Revista Médicas UIS. 2017 Nov 22;30(3):4554. https://doi.org/10.18273/revmed.v30n2-2017005

13. Llatas M. Hallazgos ecocardiográficos en pacientes seropositivos con virus de inmunodeficiencia humana, del programa TARGA del Hospital General de Jaén [tesis]. [Perú]: Universidad Nacional de Cajamarca; 2017. Disponible en http://repositorio.unc.edu.pe/handle/UNC/1183

14. Gómez M, Gómez E. Alteraciones cardiovasculares en pacientes con infección por VIH. Rev Cubana Med. $2010 \quad$ Julio-Septiembre; $49(2)$ : 268-274. $\quad$ Disponible en http://scielo.sld.cu/scielo.php?script=sci arttext\&pid=S0034-75232010000300006

15. Ponikowski P, Voors A, Anker S, Bueno H, Cleland J, Coats A et al. Guía ESC 2016 sobre el diagnóstico y tratamiento de la insuficiencia cardiaca aguda y crónica. Revista Española de Cardiología. 2016 Dec; 69(12): 1119-1125. https://doi.org/10.1016/i.recesp.2016.09.056

16. Steverson A, Pawlowski A, Schneider D, Nannapaneni P, Sanders J, Achenbach C, et al. Clinical characteristics of HIV-infected patients with adjudicated heart failure. Eur J Prev Cardiol. 2017 Noviembre; 24(16): 1746-1758. https://doi.org/10.1177/2047487317732432

17. Mouras P, Barcán L, Belziti C, Pizarro R, Ma-ez N, Marenchino R. Trasplante cardíaco en paciente infectado con el virus de la inmunodeficiencia humana. Medicina Buenos Aires. 2017 Diciembre; 77(6): 509-551. Disponible en: https://www.medicinabuenosaires.com/indices-de-2010-a-2017/volumen-77-ano2017-no-6-indice/trasplante-cardiaco-en-paciente-infectado-con-el-virus-de-la-inmunodeficiencia-humana/ 